\title{
A NUMERICAL ITERATIVE SCHEME FOR COMPUTING FINITE ORDER RANK-ONE CONVEX ENVELOPES
}

\author{
XIN WANG, ZHIPING LI ${ }^{\dagger}$ \\ LMAM \& SCHOOL OF MATHEMATICAL SCIENCES, \\ PEKING UNIVERSITY, BEIJING 100871, P.R.CHINA
}

\begin{abstract}
It is known that the $i$-th order laminated microstructures can be resolved by the $k$-th order rank-one convex envelopes with $k \geq i$. So the requirement of establishing an efficient numerical scheme for the computation of the finite order rank-one convex envelopes arises. In this paper, we develop an iterative scheme for such a purpose. The 1-st order rank-one convex envelope $R_{1} f$ is approximated by evaluating its value on matrixes at each grid point in $R^{m n}$ and then extend to non-grid points by interpolation. The approximate $k$-th order rank-one convex envelope $R_{k} f$ is obtained iteratively by computing the approximate 1-st order rank-one convex envelope of the numerical approximation of $R_{k-1} f$. Compared with $O\left(h^{1 / 3}\right)$ obtained so far for other methods, the optimal convergence rate $O(h)$ is established for our scheme, and numerical examples illustrate the computational efficiency of the scheme.
\end{abstract}

\section{INTRODUCTION}

In recent years, many amazing mechanical properties of crystalline materials such as the shape memory effect, have been successfully explained by analyzing microstructures. Ball and James developed a mathematical theory in the framework of nonlinear elasticity in which the experimentally observed geometries arise naturally as minimizers of a non-convex free energy functional [2, 3]. The theory

2000 Mathematics Subject Classification. 65K10,65N30,49A10.

Key words and phrases. finite order rank-one convex envelope, laminated microstructure, iterative scheme, interpolation.

The research was supported in part by the Special Funds for Major State Basic Research Projects (2005CB321701), NSFC projects (10431050, 10571006 and 10528102) and RFDP of China.

$\dagger$ Corresponding author: Zhiping Li, email address: lizp@math.pku.edu.cn. 
leads to the consideration of the problem of minimizing an integral functional

$$
I(u ; \Omega)=\int_{\Omega} f(x, u(x), \nabla u(x)) \mathrm{d} x
$$

with a non-quasiconvex integrand $f: \Omega \times R^{m} \times R^{m n} \rightarrow R$ in a set of admissible functions

$$
A\left(u_{0} ; \Omega\right)=\left\{u \in W^{1, p}\left(\Omega ; R^{m}\right): u=u_{0} \text { on } \partial \Omega\right\},
$$

where $\Omega \in R^{n}$ is a bounded open set with a Lipschitz continuous boundary $\partial \Omega$ and $1<p<\infty$. Since the integrand fails to be quasiconvex and hence $I(\cdot)$ is not sequentially weakly lower semicontinuous [5]. Minimizing sequences can develop oscillations, and converge weakly to a deformation which is not an energy minimizer of $I(\cdot)$, but instead an minimizer of the relaxed energy defined by

$$
I^{q c}(u ; \Omega)=\int_{\Omega} Q f(x, u(x), \nabla u(x)) \mathrm{d} x,
$$

where $Q f$ is the quasiconvex envelope of $f$, and it is known that the infimum of $I(\cdot)$ equals to the minimum of relaxed energy $I^{q}(\cdot)$. Generally it is extremely difficult if not impossible to compute $Q f$ explicitly and it is equally hard to compute $I^{q c}$. However, it is known that the $i$-th order laminated microstructures, or laminates in laminates, can be solved by any of the $k$-th order rank-one convex envelopes with $k \geq i$ [10]. So the requirement of establishing an efficient numerical scheme for the computation of finite order rank-one convex envelopes arises. Great progress on numerical analysis and methods for rank-one convex envelopes has been made in recent years $[1,6,7,8,10,11]$

In the present paper, a new approach for computing the finite order rank-one convex envelope is given. $R_{1} f(A)$ is expressed as a unconstrained optimization problem with $(m+n)$ variables as in [10] and the 1-st order rank-one convex envelope $R_{1} f$ is approximated by evaluating its value on matrixes at each grid point in $R^{m n}$ and then extend to non-grid points by interpolation. The approximate $k$-th order rank-one convex envelope $R_{k} f$ is obtained iteratively by computing the approximate 1-st order rank-one convex envelope of the numerical approximation of $R_{k-1} f$. In Section 2, the definition and properties of finite order rank-one convex envelopes are presented. In Section 3, the new scheme is established and analyzed, compared with the convergence rate of $O\left(h^{1 / 3}\right)$ for Dolzmann's method [7] and similar results for finite element method [4], the optimal convergence rate of 
$O(h)$ is obtained for our scheme. In Section 4, numerical examples are presented to illustrate the computational efficiency of the scheme.

\section{Definition and properties of $R_{k} f$}

We first introduce the definition of $R_{k} f$. Let $f: R^{m n} \rightarrow R^{1} \cup\{\infty\}$ be continuous.

Definition 2.1. Let $R_{1} f: R^{m n} \rightarrow R^{1} \cup\{\infty\}$ be defined by

$$
\begin{aligned}
& R_{1} f(A)=\inf \left\{\lambda f\left(A_{0}\right)+(1-\lambda) f\left(A_{1}\right): 0 \leq \lambda \leq 1,\right. \\
&\left.A=\lambda A_{0}+(1-\lambda) A_{1}, \operatorname{rank}\left(A_{0}-A_{1}\right) \leq 1\right\},
\end{aligned}
$$

and let $R_{k} f: R^{m n} \rightarrow R^{1} \cup\{\infty\}$ be defined by

$$
R_{k} f(A)=R_{1}\left(R_{k-1} f\right)(A) .
$$

$R_{k} f$ is called the $k$-th order rank-one convex envelope of $f$.

Lemma 2.1. $[9,10]$ The finite order rank-one convex envelopes have the following properties:

(1): $\lim _{k \rightarrow \infty} R_{k} f(A)=R f(A), \forall A \in R^{m n}$;

(2): $f \geq R_{1} f \geq R_{2} f \geq \cdots \geq R_{k} f \geq \cdots \geq R f$,

where $R f$ is the so called rank-one convex envelope of the function $f$.

Our method for the computation of the finite order rank-one convex envelope is based on an efficient scheme to compute $R_{1} f(A)$, for which we follow the approach of $\operatorname{Li}[10]$. Let $A \in R^{m n}$, suppose that we have

$$
A=\lambda A_{0}+(1-\lambda) A_{1}, \quad \operatorname{rank}\left(A_{0}-A_{1}\right) \leq 1,
$$

then, there exist $\theta \in S^{m-1}, \phi \in S^{n-1}, \gamma \in R^{1}, \tau \in S^{1}$ such that

$$
A_{0}=A+\gamma \sin ^{2}(\tau) \phi \otimes \theta, \quad A_{1}=A-\gamma \cos ^{2}(\tau) \phi \otimes \theta,
$$

where $\tau=\arcsin (\sqrt{1-\lambda})$ and $S^{i-1}$ is the unit sphere in $R^{i}$ centered at 0 .

Thus, to compute $R_{1} f(A)$ is equivalent to solve a nonlinear unconstrained optimization problem with $(m+n)$ variables. The first order rank-one envelope can be written as

$$
R_{1} f(A)=\inf \left\{\cos ^{2}(\tau) f\left(A+\gamma \sin ^{2}(\tau) \phi \otimes \theta\right)+\sin ^{2}(\tau) f\left(A-\gamma \cos ^{2}(\tau) \phi \otimes \theta\right)\right\},
$$


where the infimum is taken over $\theta \in S^{m-1}, \phi \in S^{n-1}, \gamma \in R^{1}, \tau \in S^{1}$.

Next we discuss the relationship between $f$ and $R_{1} f$. Denote

$$
D_{1}=\left\{(\theta, \phi, \tau, \gamma) \in S^{n-1} \times S^{m-1} \times S^{1} \times R^{1}\right\} .
$$

For $\delta_{1} \in D_{1}$, define

$$
f^{1}\left(A ; \delta_{1}\right)=\cos ^{2}(\tau) f\left(A+\gamma \sin ^{2}(\tau) \phi \otimes \theta\right)+\sin ^{2}(\tau) f\left(A-\gamma \cos ^{2}(\tau) \phi \otimes \theta\right) .
$$

Then the first order rank-one convex envelope can be written as

$$
R_{1} f(A)=\inf _{\delta_{1} \in D_{1}} f^{1}\left(A ; \delta_{1}\right)
$$

The following two lemmas are given by Li in [11].

Lemma 2.2. [11] Let $f: R^{m n} \rightarrow R^{1} \cup\{\infty\}$ be continuous and satisfy

$$
(H 1): f(\cdot) \text { is bounded from below and } \frac{f(B)}{\|B\|} \rightarrow \infty \text { as }\|B\| \rightarrow \infty,
$$

then, for any given $A \in R^{m n}$ and integer $k \geq 1$, there exist $\delta_{1}(A) \in D_{1}$ such that

$$
f^{1}\left(A, \delta_{1}(A)\right)=\inf _{\delta_{1} \in D_{1}} f^{1}\left(A ; \delta_{1}\right) .
$$

Lemma 2.3. [11] If $f(\cdot)$ is continuous and satisfy $(H 1)$, then its first order rank-one convex envelope $R_{1} f(\cdot)$ is also continuous and satisfy $(H 1)$.

In fact, we can prove the following lemma.

Lemma 2.4. If $f(\cdot)$ is locally Lipschitz continuous and satisfies $(H 1)$, then its first order rank-one convex envelope $R_{1} f(\cdot)$ is also locally Lipschitz continuous.

Proof. Let $A, B \in R^{m n}$, since $f(\cdot)$ is locally Lipschitz continuous, there exists an non-decreasing function $c:[0, \infty) \rightarrow R_{+}^{1}$, such that

$$
|f(A)-f(B)| \leq c(\|A\|+\|B\|)|A-B| .
$$

By lemma 2.2, there exist $\delta_{1} \in D_{1}$ and $\delta_{2} \in D_{1}$ such that

$$
\begin{aligned}
& R_{1} f(A)=\cos ^{2}\left(\tau_{1}\right) f\left(A+\gamma_{1} \sin ^{2}\left(\tau_{1}\right) \phi_{1} \otimes \theta_{1}\right)+\sin ^{2}\left(\tau_{1}\right) f\left(A-\gamma_{1} \cos ^{2}\left(\tau_{1}\right) \phi_{1} \otimes \theta_{1}\right), \\
& R_{1} f(B)=\cos ^{2}\left(\tau_{2}\right) f\left(B+\gamma_{2} \sin ^{2}\left(\tau_{2}\right) \phi_{2} \otimes \theta_{2}\right)+\sin ^{2}\left(\tau_{2}\right) f\left(B-\gamma_{2} \cos ^{2}\left(\tau_{2}\right) \phi_{2} \otimes \theta_{2}\right) .
\end{aligned}
$$


By $(\mathrm{H} 1)$, there exists a non-decreasing function $c_{2}:[0, \infty) \rightarrow R_{+}^{1}$, such that $\max \left\{\left|\gamma_{1}\right|,\left|\gamma_{2}\right|\right\} \leq c_{2}(\|A\|+\|B\|)$. Let

$R_{1} \bar{f}(A)=\cos ^{2}\left(\tau_{2}\right) f\left(A+\gamma_{2} \sin ^{2}\left(\tau_{2}\right) \phi_{2} \otimes \theta_{2}\right) \sin ^{2}\left(\tau_{2}\right) f\left(A-\gamma_{2} \cos ^{2}\left(\tau_{2}\right) \phi_{2} \otimes \theta_{2}\right)$,

$R_{1} \bar{f}(B)=\cos ^{2}\left(\tau_{1}\right) f\left(B+\gamma_{1} \sin ^{2}\left(\tau_{1}\right) \phi_{1} \otimes \theta_{1}\right)+\sin ^{2}\left(\tau_{1}\right) f\left(B-\gamma_{1} \cos ^{2}\left(\tau_{1}\right) \phi_{1} \otimes \theta_{1}\right)$.

It follows from $R_{1} f(A) \leq R_{1} \bar{f}(A)$ and the local Lipschitz continuity of $f$ that

$$
\begin{aligned}
& R_{1} f(A)-R_{1} f(B)=R_{1} f(A)-R_{1} \bar{f}(A)+R_{1} \bar{f}(A)-R_{1} f(B) \\
\leq & R_{1} \bar{f}(A)-R_{1} f(B) \\
= & \cos ^{2}\left(\tau_{2}\right)\left\{f\left(A+\gamma_{2} \sin ^{2}\left(\tau_{2}\right) \phi_{2} \otimes \theta_{2}\right)-f\left(B+\gamma_{2} \sin ^{2}\left(\tau_{2}\right) \phi_{2} \otimes \theta_{2}\right)\right\} \\
& +\sin ^{2}\left(\tau_{2}\right)\left\{f\left(A-\gamma_{2} \cos ^{2}\left(\tau_{2}\right) \phi_{2} \otimes \theta_{2}\right)-f\left(B-\gamma_{2} \cos ^{2}\left(\tau_{2}\right) \phi_{2} \otimes \theta_{2}\right)\right\} \\
\leq & c\left(\|A\|+\|B\|+c_{2}(\|A\|+\|B\|)\right)|A-B| .
\end{aligned}
$$

On the other hand, it follows from $R_{1} \bar{f}(B) \geq R_{1} f(B)$ and the local Lipschitz continuity of $f$ that

$$
\begin{aligned}
R_{1} f(A)-R_{1} f(B) & =R_{1} f(A)-R_{1} \bar{f}(B)+R_{1} \bar{f}(B)-R_{1} f(B) \\
& \geq R_{1} f(A)-R_{1} \bar{f}(B) \\
& \geq-c\left(\|A\|+\|B\|+c_{2}(\|A\|+\|B\|)\right)|A-B| .
\end{aligned}
$$

This completes the proof.

We can further show that, as an operator mapping $C\left(R^{m n}\right)$ on to $C\left(R^{m n}\right), R_{1}$ is also Lipschitz continuous with corresponding Lipschitz constant $c=1$. More precisely we have the following lemma.

Lemma 2.5. If $f, g$ are continuous and satisfy $(H 1)$, then we have $\| R_{1} f-$ $R_{1} g\left\|_{\infty} \leq\right\| f-g \|_{\infty}$.

Proof. For any $A \in R^{m n}$, since $f, g$ are continuous and satisfy (H1), by Lemma 2.2, there exist $\delta_{1} \in D_{1}, \delta_{2} \in D_{1}$ such that

$$
\begin{aligned}
& R_{1} f(A)=\cos ^{2}\left(\tau_{1}\right) f\left(A+\gamma_{1} \sin ^{2}\left(\tau_{1}\right) \phi_{1} \otimes \theta_{1}\right)+\sin ^{2}\left(\tau_{1}\right) f\left(A-\gamma_{1} \cos ^{2}\left(\tau_{1}\right) \phi_{1} \otimes \theta_{1}\right), \\
& R_{1} g(A)=\cos ^{2}\left(\tau_{2}\right) g\left(A+\gamma_{2} \sin ^{2}\left(\tau_{2}\right) \phi_{2} \otimes \theta_{2}\right)+\sin ^{2}\left(\tau_{2}\right) g\left(A-\gamma_{2} \cos ^{2}\left(\tau_{2}\right) \phi_{2} \otimes \theta_{2}\right) .
\end{aligned}
$$


Exchange $\delta_{1}$ and $\delta_{2}$ in the definitions of $R_{1} f(A)$ and $R_{1} g(A)$, and denote

$R_{1} \bar{f}(A)=\cos ^{2}\left(\tau_{2}\right) f\left(A+\gamma_{2} \sin ^{2}\left(\tau_{2}\right) \phi_{2} \otimes \theta_{2}\right)+\sin ^{2}\left(\tau_{2}\right) f\left(A-\gamma_{2} \cos ^{2}\left(\tau_{2}\right) \phi_{2} \otimes \theta_{2}\right)$,

$R_{1} \bar{g}(A)=\cos ^{2}\left(\tau_{1}\right) g\left(A+\gamma_{1} \sin ^{2}\left(\tau_{1}\right) \phi_{1} \otimes \theta_{1}\right)+\sin ^{2}\left(\tau_{1}\right) g\left(A-\gamma_{1} \cos ^{2}\left(\tau_{1}\right) \phi_{1} \otimes \theta_{1}\right)$.

Since $R_{1} f(A) \leq R_{1} \bar{f}(A)$, we have

$$
\begin{aligned}
& R_{1} f(A)-R_{1} g(A)=R_{1} f(A)-R_{1} \bar{f}(A)+R_{1} \bar{f}(A)-R_{1} g(A) \\
\leq & R_{1} \bar{f}(A)-R_{1} g(A) \\
= & \cos ^{2}\left(\tau_{2}\right)\left\{f\left(A+\gamma_{2} \sin ^{2}\left(\tau_{2}\right) \phi_{2} \otimes \theta_{2}\right)-g\left(A+\gamma_{2} \sin ^{2}\left(\tau_{2}\right) \phi_{2} \otimes \theta_{2}\right)\right\} \\
& +\sin ^{2}\left(\tau_{2}\right)\left\{f\left(A-\gamma_{2} \cos ^{2}\left(\tau_{2}\right) \phi_{2} \otimes \theta_{2}\right)-g\left(A-\gamma_{2} \cos ^{2}\left(\tau_{2}\right) \phi_{2} \otimes \theta_{2}\right)\right\} \\
\leq & \|f-g\|_{\infty} .
\end{aligned}
$$

On the other hand, since $R_{1} \bar{g}(A) \geq R_{1} g(A)$, we have

$$
\begin{aligned}
& R_{1} f(A)-R_{1} g(A)=R_{1} f(A)-R_{1} \bar{g}(A)+R_{1} \bar{g}(A)-R_{1} g(A) \\
\geq & R_{1} f(A)-R_{1} \bar{g}(A) \\
= & \cos ^{2}\left(\tau_{1}\right)\left\{f\left(A+\gamma_{1} \sin ^{2}\left(\tau_{1}\right) \phi_{1} \otimes \theta_{1}\right)-g\left(A+\gamma_{1} \sin ^{2}\left(\tau_{1}\right) \phi_{1} \otimes \theta_{1}\right)\right\} \\
& +\sin ^{2}\left(\tau_{1}\right)\left\{f\left(A-\gamma_{1} \cos ^{2}\left(\tau_{1}\right) \phi_{1} \otimes \theta_{1}\right)-g\left(A-\gamma_{1} \cos ^{2}\left(\tau_{1}\right) \phi_{1} \otimes \theta_{1}\right)\right\} \\
\geq & -\|f-g\|_{\infty} .
\end{aligned}
$$

Thus, we have

$$
\left|R_{1} f(A)-R_{1} g(A)\right| \leq\|f-g\|_{\infty}, \quad \forall A \in R^{m n} .
$$

This yields the conclusion of the lemma.

The above results on $R_{1} f$ can be easily extend to $R_{k} f$.

Corollary 2.1. If $f(\cdot)$ is Lipschitz continuous and satisfy $(H 1)$, then its finite order rank-one convex envelope $R_{k} f(\cdot)$, for $k=1,2, \ldots$, are all Lipschitz continuous with the same Lipschitz constant and satisfy (H1).

Proof. The conclusion of the corollary follows directly, by induction, from the recursive definition of $R_{k} f$, Lemma 2.3 and Lemma 2.4.

The next result was given by Dolzmann. 
Lemma 2.6. [7] Assume that $f, g^{r c}: R^{m n} \rightarrow R$ are continuous and $f \geq g^{r c}$ on $R^{m n}, f=g^{r c}$ on $R^{m n} \backslash B_{r}(0)$ and $g^{r c}$ is rank-one convex, where $B_{r}(0)=\{A \in$ $\left.R^{m n} ;\|A\|_{\infty} \leq r\right\}$. Define $\tilde{f}: R^{m n} \rightarrow R$ by

$$
\widetilde{f}(A):=\left\{\begin{array}{c}
\inf \left\{\sum_{i=1}^{N} \lambda_{i} f\left(A_{i}\right):\left(\lambda_{i}, A_{i}\right) \in H_{N}, A_{i} \in B_{r}(0),\right. \\
\left.\quad \text { and } A=\sum_{i=1}^{N} \lambda_{i} A_{i}\right\}, \quad \text { if } A \in B_{r}(0), \\
f(A), \quad \text { if } A \in R^{m n} \backslash B_{r}(0) .
\end{array}\right.
$$

Then $\tilde{f}=R f$.

We can show that a similar result holds for $R_{k} f$.

Theorem 2.1. Assume that $f, g^{r c}: R^{m n} \rightarrow R$ are continuous and $f \geq g^{r c}$ on $R^{m n}, f=g^{r c}$ on $R^{m n} \backslash B_{r}(0)$ and $g^{r c}$ is rank-one convex, where again $B_{r}(0)=$ $\left\{A \in R^{m n} ;\|A\|_{\infty} \leq r\right\}$. Define $\tilde{f}_{k}: R^{m n} \rightarrow R$ by

$$
\widetilde{f}_{k}(A):= \begin{cases}R_{k} f(A), & \text { if } A \in B_{r}(0), \\ f(A), & \text { if } A \in R^{m n} \backslash B_{r}(0) .\end{cases}
$$

Then $\widetilde{f}_{k}=R_{k} f$.

Proof. By Lemma 2.6, we know that $\widetilde{f}_{k}(A):=f(A)=R f(A)$ for all $A \in R^{m n} \backslash$ $B_{r}(0)$, while by Lemma 2.1 , we have $\widetilde{f}_{k} \geq R_{k} f \geq R f$. Thus, we have $\widetilde{f}_{k}(A)=$ $R_{k} f(A)$ for all $A \in R^{m n} \backslash B_{r}(0)$, this together with the definition of $\widetilde{f}_{k}(A)$ implies that the conclusion of the theorem holds.

Remark 2.1. With the above theorem, in our numerical computation of $R_{k} f$, if there exists a rank-one convex function $g^{r c}$, such that $f \geq g^{r c}$ on $B_{r}(0)$ and $f=g^{r c}$ on $R^{m n} \backslash B_{r}(0)$, then we only need to compute $R_{k} f$ on $B_{r}(0)$. We remark also that when $B_{r}(0)$ is replaced by any convex set in $R^{m n}$ Lemma 2.6 and hence Theorem 2.1 still hold.

\section{The SCHEME For COMPUTING $R_{k} f$}

First we introduce the definition for the finite order approximate rank-one convex envelope. 
Definition 3.1. Let $\triangle_{h_{i}}=h_{i} Z^{m \times n}$ be a set of uniform grids on $R^{m n}$, where $Z$ is the set of all integers and $\left\{h_{i}\right\}_{i=1}^{k}$ is a given set of mesh size. Define the 1-st order approximate rank-one convex operator $R_{1}^{h_{1}}: C\left(R^{m n} ; R \cup\{\infty\}\right) \rightarrow$ $C\left(R^{m n} ; R \cup\{\infty\}\right)$ by

$$
R_{1}^{h_{1}} f(A)= \begin{cases}R_{1} f(A), & \text { if } A \in \triangle_{h_{1}}, \\ Q_{m n}\left(R_{1} f\left(\triangle_{h_{1}}\right)\right)(A), & \text { otherwise, }\end{cases}
$$

where $Q_{m n}\left(R_{1} f\left(\triangle_{h_{1}}\right)\right)(A)$ means to evaluate by $m \times n$ multi-linear interpolation using the grid values $R_{1} f\left(\triangle_{h_{1}}\right)$. We call $R_{1}^{h_{1}} f$ the 1 -st order approximate rankone convex envelope of $f$.

The $k$-th order approximate rank-one convex envelope of a function $f$ is defined recursively by

$$
R_{k}^{h_{k}} f(A)= \begin{cases}R_{1}\left(R_{k-1}^{h_{k-1}} f\right)(A), & \text { if } A \in \triangle_{h_{k}} \\ Q_{m n}\left[R_{1}\left(R_{k-1}^{h_{k-1}} f\right)\left(\triangle_{h_{k}}\right)\right](A), & \text { otherwise }\end{cases}
$$

Our scheme for the computation of the $k$-th order rank-one convex envelope $R_{k} f$ is based on the definition of the $k$-th order approximate order rank-one convex envelope $R_{k}^{h_{k}}$ :

(1): set $i:=0$ and $R_{0}^{h_{0}} f=f$;

(2): set $i:=i+1$, compute the function value of $R_{i}^{h_{i}} f$ on $\triangle_{h_{i}}$ by using $R_{i}^{h_{i}} f(A):=R_{1}^{h_{i}}\left(R_{i-1}^{h_{i-1}} f\right)(A)$, for $A \in \triangle_{h_{i}} ;$

(3): if $i>=k$, output the grid value $R_{j}^{h_{j}} f\left(\triangle_{h_{j}}\right), j=1,2, \ldots, k$, and stop; else goto (2).

We have the following result for the error of the approximation.

Theorem 3.1. Let $f$ satisfy the hypothesis (H1) and be Lipschitz continuous on $R^{m n}$ with Lipschitz constant L, let the mesh sizes $\left\{h_{i}\right\}_{i=1}^{k}$ be given. Then, (a): $R_{k}^{h_{k}} f$ satisfies the hypothesis (H1) and is Lipschitz continuous on $R^{m n}$ with the same Lipschitz constant L; (b): Further more, we have the following error estimate

$$
\sup _{A \in R^{m n}}\left|R_{k} f(A)-R_{k}^{h_{k}} f(A)\right| \leq L \sum_{i=1}^{k} h_{i}
$$


Proof. We shall prove the theorem by induction.

(1): For $k=1$, by Lemma 2.3 and Lemma 2.4, $R_{1} f$ satisfies $(H 1)$ and is Lipschitz continuous on $R^{m n}$ with Lipschitz constant $L$. Thus, by (3.1), it is easily seen that, as a multilinear interpolation of $R_{1} f$ on the grid $\triangle_{h_{1}}, R_{1}^{h_{1}} f$ also satisfies $(H 1)$ and is Lipschitz continuous with the same Lipschitz constant $L$, that is $(a)$ holds for $k=1$. Since the mesh size for the multilinear interpolation is $h_{1}$, we have

$$
\sup _{A \in R^{m n}}\left|R_{1} f(A)-R_{1}^{h_{1}} f(A)\right|=\sup _{A \in R^{m n}}\left|R_{1} f(A)-Q_{m n}\left(R_{1} f\left(\triangle_{h_{1}}\right)\right)(A)\right| \leq L h_{1} .
$$

This shows that $(b)$ holds for $k=1$.

(2): Now, assume that the theorem holds for $1 \leq k \leq j-1$, that is for all $1 \leq k \leq j-1,(a)$ holds, and we have

$$
\sup _{A \in R^{m n}}\left|R_{k} f(A)-R_{k}^{h_{k}} f(A)\right| \leq L \sum_{i=1}^{k} h_{i}, \quad \forall k \in\{1,2 \ldots, j-1\} .
$$

Again by Lemma 2.3 and Lemma 2.4 and by the induction assumption, $R_{1}\left(R_{j-1}^{h_{j-1}} f\right)$ satisfies $(a)$. Thus, by (3.2) with $k=j$ and (3.4), and by Lemma 2.4 and Lemma 2.5, we have

$$
\begin{aligned}
\left|R_{j} f(A)-R_{j}^{h_{j}} f(A)\right| & \leq\left|R_{1}\left(R_{j-1} f\right)(A)-R_{1}\left(R_{j-1}^{h_{j-1}} f\right)(A)\right| \\
& +\left|R_{1}\left(R_{j-1}^{h_{j-1}} f\right)(A)-Q_{m n}\left[R_{1}\left(R_{j-1}^{h_{j-1}}\right) f\left(\triangle_{h_{j}}\right)\right](A)\right| \\
& \leq\left|R_{j-1} f(A)-R_{j-1}^{h_{j-1}} f(A)\right|+L h_{j} \leq L \sum_{i=1}^{j} h_{i} .
\end{aligned}
$$

The theorem follows now from the induction principle.

Remark 3.1. Compared with the result of Dolzmann[7] where the convergence rate of $O\left(h^{1 / 3}\right)$ is shown to hold for the method established therein and similar results elsewhere [4], the convergence rate of our method reaches $O(h)$. It is easily seen that $O(h)$ is the optimal convergence rate for the computation of the finite order rank-one convex envelope, if $f$ is only required to be Lipschitz continuous. However, if a higher order interpolation is used near the smooth points of $R_{i} f$ involved in the computation, then we might expect to obtain higher 
order approximation locally, as is seen in the proof of Theorem 3.1 that the interpolation error is what actually counts.

\section{NumERICAL EXPERIMENTS}

Example 1. Let $f: R^{2 \times 2} \rightarrow R$ be given by [4]

$$
f(A)=\prod_{i=0}^{3}\left\|A-A_{i}\right\|_{F} .
$$

where $\left\|\left(a_{i, j}\right)\right\|_{F}=\left(\sum_{i, j=1}^{2} a_{i, j}^{2}\right)^{1 / 2}$ is the Frobenius norm of the matrix in $R^{2 \times 2}$, and

$$
\begin{aligned}
A_{0}=\left(\begin{array}{cc}
-1 & 0 \\
0 & -1
\end{array}\right), & A_{1}=\left(\begin{array}{cc}
-1 & 0 \\
0 & 1
\end{array}\right), \\
A_{2}=\left(\begin{array}{ll}
1 & 0 \\
0 & 1
\end{array}\right), & A_{3}=\left(\begin{array}{cc}
1 & 0 \\
0 & -1
\end{array}\right) .
\end{aligned}
$$

It is easily seen that $f$ is a non-negative function with 4 zero points $A_{i}, i=$ $0,1,2,3$. Figure 1 shows the graph of $f$ in the $a_{11}-a_{22}$ plane.

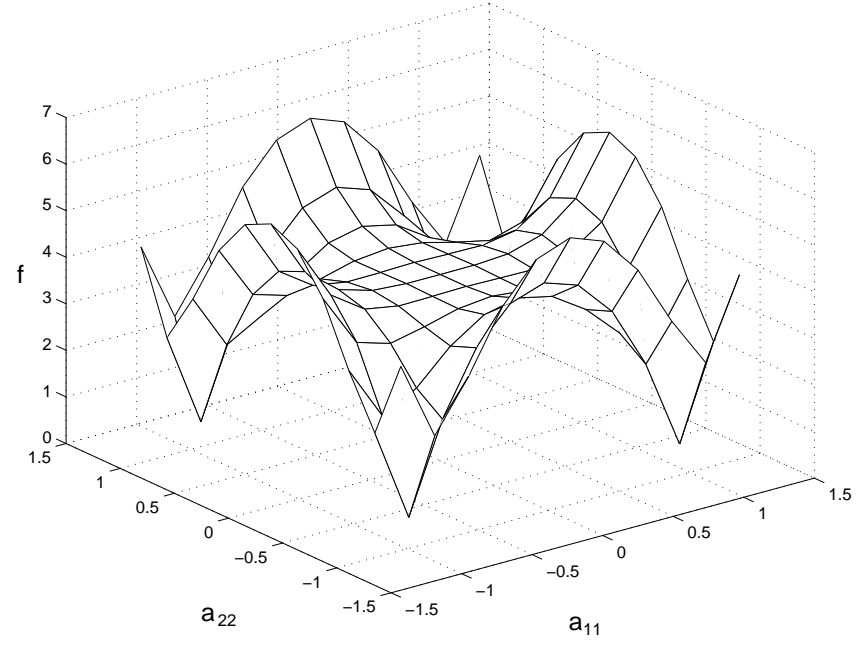

Figure 1. $f$ on $[-1.1,1.1] \times[-1.1,1.1]$ in $a_{11}-a_{22}$ plane.

Since the matrixes $\left(A_{0}-A_{1}\right),\left(A_{1}-A_{2}\right),\left(A_{2}-A_{3}\right)$ and $\left(A_{3}-A_{0}\right)$ are rank-

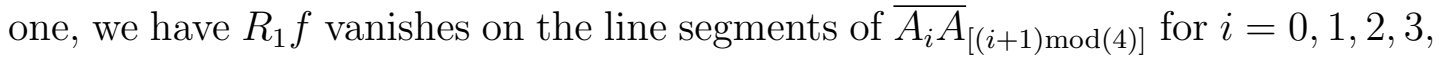


where $[(i+1) \bmod (4)]=i+1$ for $i=0,1,2$, and $[(3+1) \bmod (4)]=0$, that is

$$
R_{1} f(A)=0, \quad \text { if } A=\lambda A_{i}+(1-\lambda) A_{(i+1) \bmod (4)}, i=0,1,2,3, \forall \lambda \in[0,1] .
$$

Figure 2 shows the rank-one connected line segments between the zero points of $f$ in $a_{11}-a_{22}$ plane. Since the matrixes with same entries other than $a_{11}$ or $a_{22}$ are rank-one connected, (4.2) implies that

$$
R_{2} f(A)=0, \quad \text { if } A \in \mathcal{D}=\left\{D: d_{12}=d_{21}=0,\left|d_{11}\right| \leq 1 \text { and }\left|d_{22}\right| \leq 1\right\}
$$

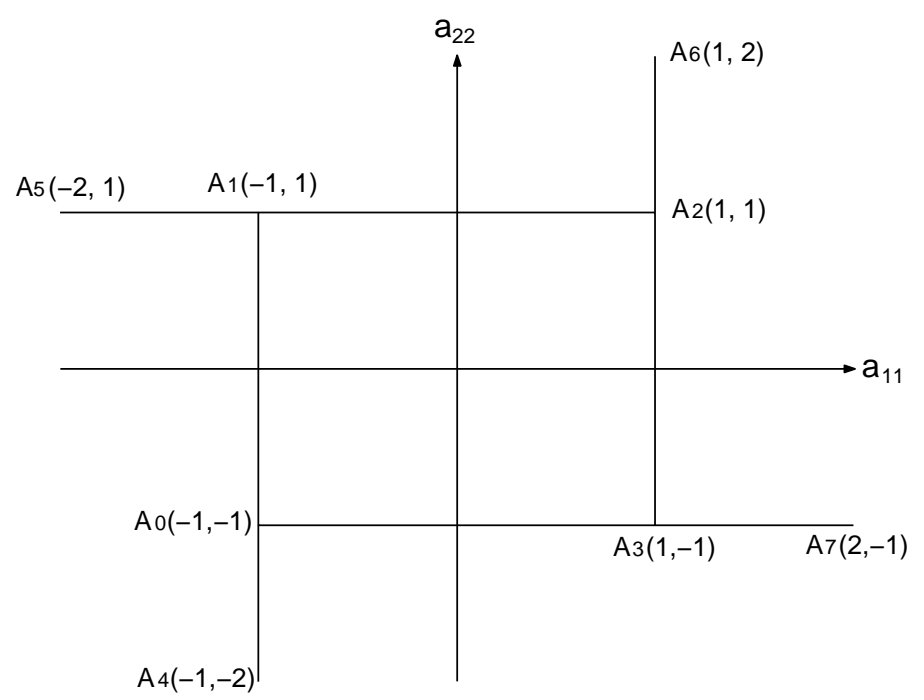

FiguRE 2. Line segments with rank-one connection in $a_{11}-a_{22}$ plane.

The numerical results of $R_{1}^{h} f$ and $R_{2}^{h} f$ for $h=0.1$ are shown in Figure 3 and Figure 4 respectively. It is clearly seen that $R_{2}^{0.1} f$ vanishes in the set $\mathcal{D}$ where $a_{12}=a_{21}=0$ and both $\left|a_{11}\right|$ and $\left|a_{22}\right|$ are no greater than 1 , in fact our numerical experiments produce the result in machine accuracy. Figure 5 shows the convergence behavior of $R_{1}^{h} f$ with respect to $h$ by showing the convergence behavior of $\left\|R_{1}^{h} f-R_{1}^{h / 2} f\right\|_{\infty}$ in the set $\mathcal{D}$ where $a_{12}=a_{21}=0$ and both $\left|a_{11}\right|$ and $\left|a_{22}\right|$ are no greater than 1. In fact, our numerical experiments show that $R_{1}^{h} f(A) \approx R_{1} f(A)+5 h$, which agrees very well with the analytical result given in Theorem 3.1. 


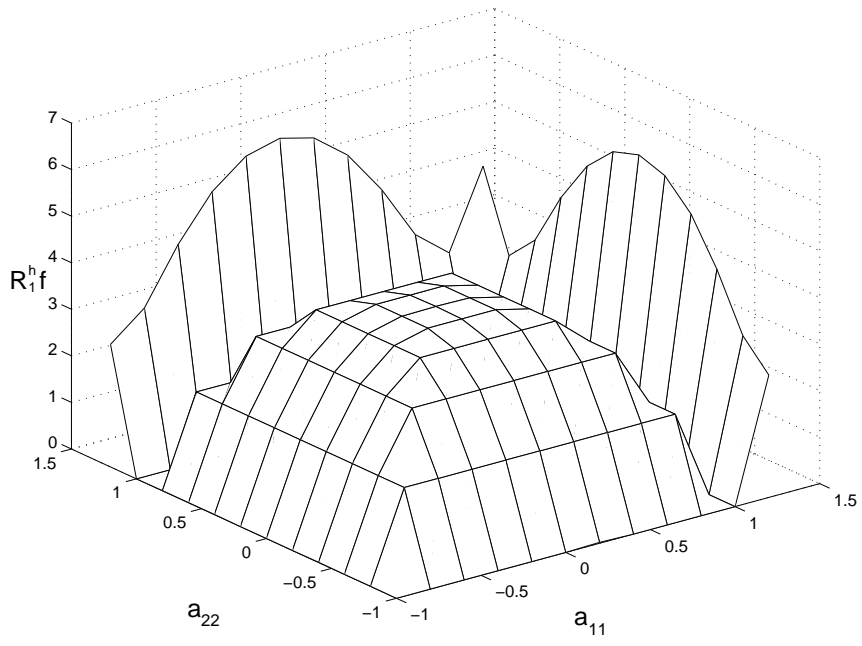

Figure $3 . R_{1}^{0.1} f$ on $[-1,1.1] \times[-1,1.1]$ in $a_{11}-a_{22}$ plane.

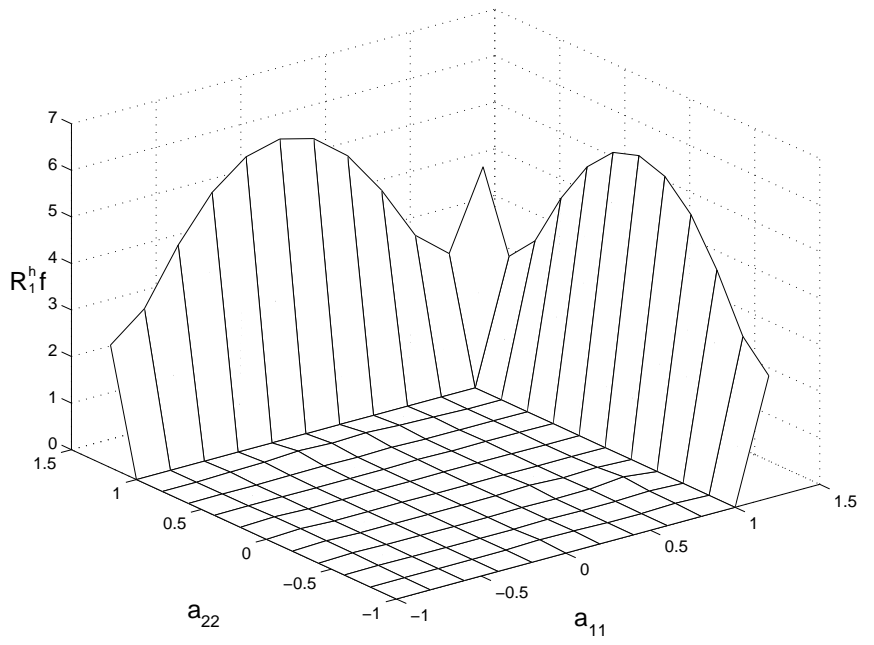

Figure $4 . R_{2}^{0.1} f$ on $[-1,1.1] \times[-1,1.1]$ in $a_{11}-a_{22}$ plane.

Example 2. Let $f: R^{2 \times 2} \rightarrow R$ be defined by [4]

$$
f(A)=\prod_{i=4}^{7}\left\|A-A_{i}\right\|_{F}
$$

where,

$$
A_{4}=\left(\begin{array}{cc}
-1 & 0 \\
0 & -2
\end{array}\right), \quad A_{5}=\left(\begin{array}{cc}
-2 & 0 \\
0 & 1
\end{array}\right)
$$




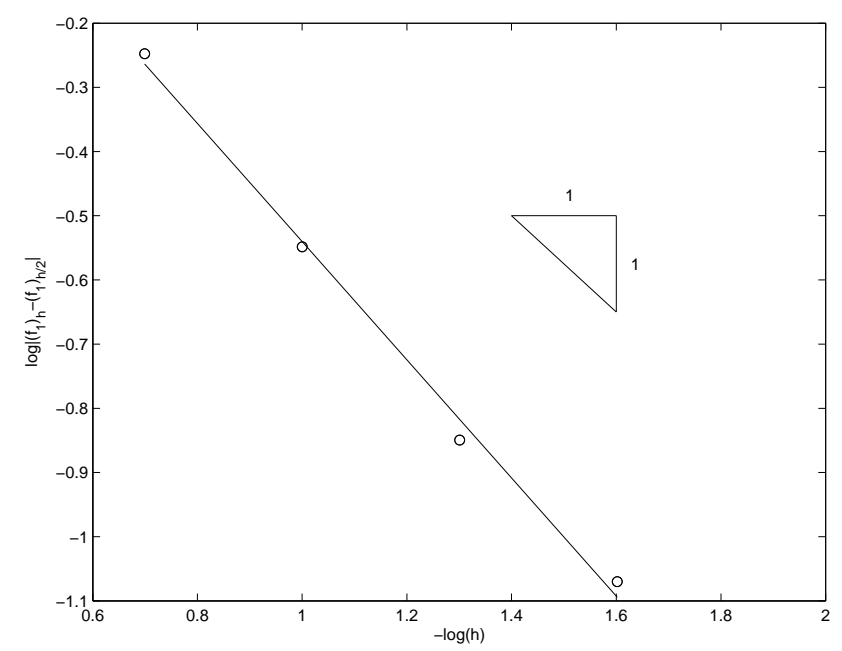

FiguRE 5. The convergence behavior of $R_{1}^{h} f$ on $[-1,1] \times[-1,1]$.

$$
A_{6}=\left(\begin{array}{ll}
1 & 0 \\
0 & 2
\end{array}\right), \quad A_{7}=\left(\begin{array}{cc}
2 & 0 \\
0 & -1
\end{array}\right)
$$

It is easily seen that $f$ is again a non-negative function but with 4 zero points moving out a little bit to $A_{i}, i=4,5,6,7$ (see Figure 2). Figure 6 shows the graph of $f$ in the $a_{11}-a_{22}$ plane.

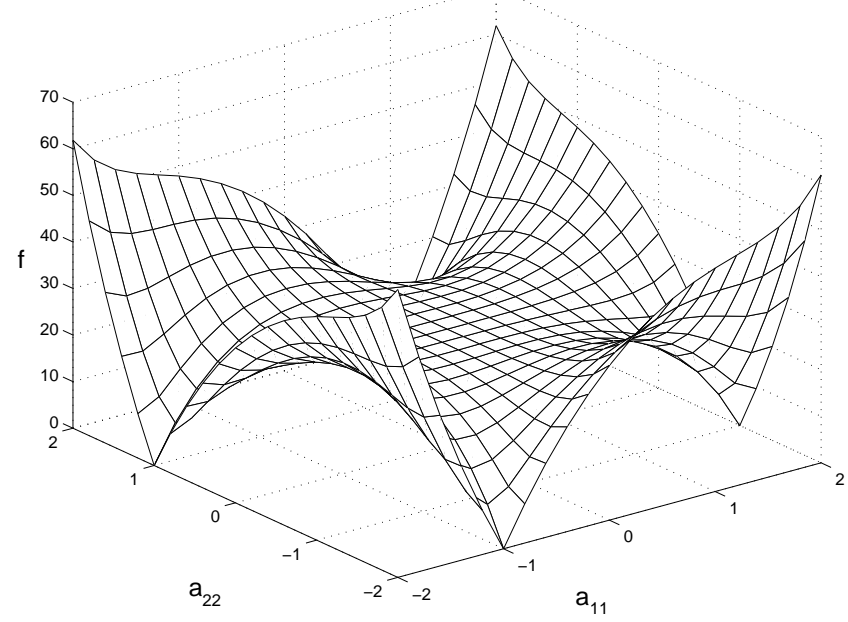

Figure $6 . f$ on $[-2,2] \times[-2,2]$ in $a_{11}-a_{22}$ plane 


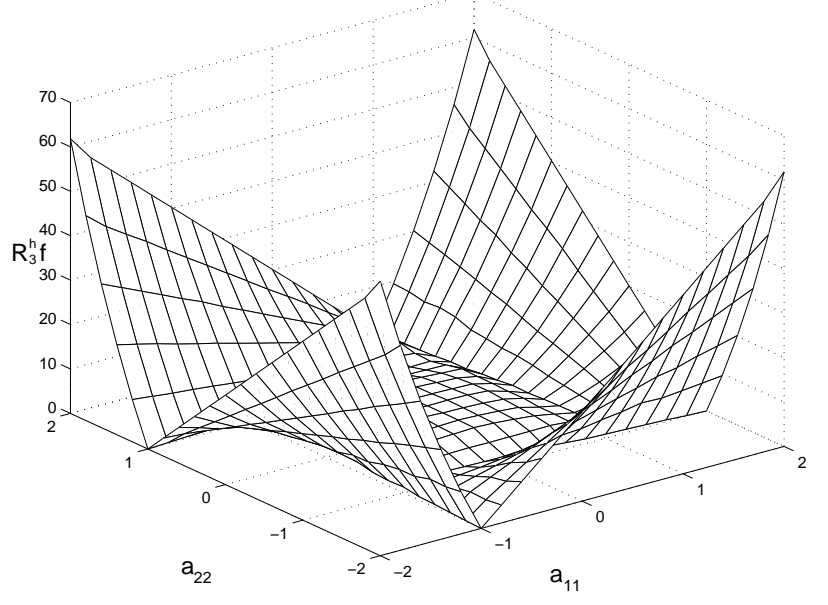

Figure $7 . R_{3} f$ on $[-2,2] \times[-2,2]$ in $a_{11}-a_{22}$ plane.

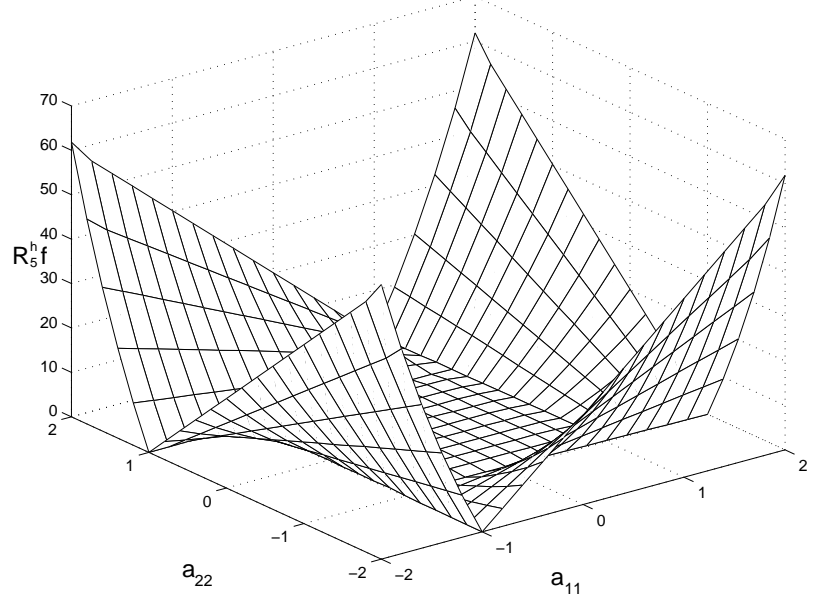

Figure $8 . R_{5} f$ on $[-2,2] \times[-2,2]$ in $a_{11}-a_{22}$ plane.

The zero points of $f$ are no longer in ran-one connection, however, we have the matrixes $\left(A_{4}-A_{0}\right),\left(A_{4}-A_{1}\right),\left(A_{5}-A_{1}\right),\left(A_{5}-A_{2}\right),\left(A_{6}-A_{2}\right),\left(A_{6}-A_{3}\right)$, $\left(A_{7}-A_{3}\right)$ and $\left(A_{7}-A_{0}\right)$ are rank-one. In Figure 2 , these rank-one connections are shown by the line segments. Unlike in the case of Example 1 , for any $k \geq 1$, $R_{k}^{h} f$ here is nonzero except at $A_{i}, i=4,5,6,7$. However, we have [7]

$$
R f(A)=0, \quad \text { if } A \in \mathcal{D}=\left\{D: d_{12}=d_{21}=0,\left|d_{11}\right| \leq 1 \text { and }\left|d_{22}\right| \leq 1\right\} .
$$

This, together with Lemma 2.1 and Theorem 3.1, implies

$$
\lim _{k \rightarrow \infty} \lim _{h \rightarrow 0} R_{k}^{h} f(A)=0, \quad \forall A \in \mathcal{D} .
$$


In Figure 7, Figure 8 and Figure 9 the numerical results of $R_{3}^{h} f(A), R_{5}^{h} f(A)$ and $R_{6}^{h} f(A)$ with $h=0.2$ are shown respectively, where it is clearly seen that, even with fixed $h$, the numerical results lead to

$$
\lim _{k \rightarrow \infty} R_{k}^{h} f(A)=0, \quad \forall A \in \mathcal{D} .
$$

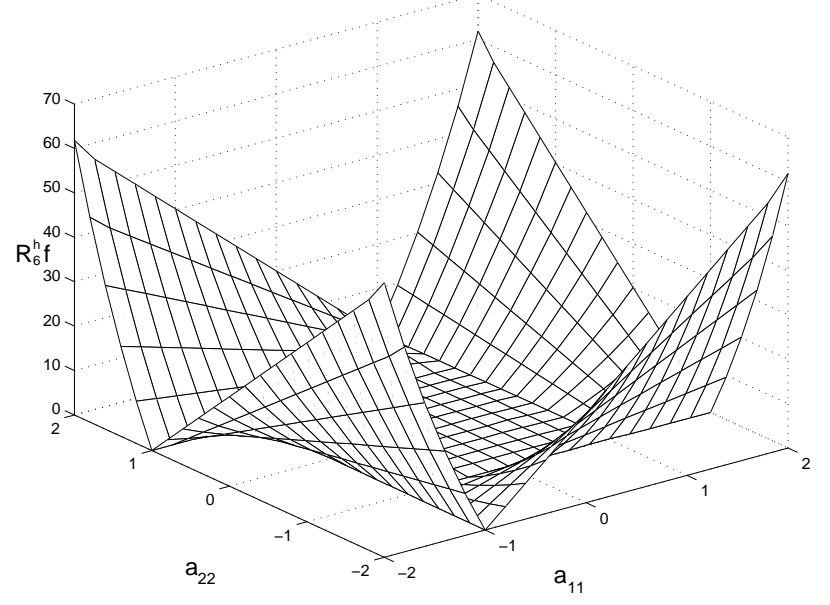

Figure $9 . R_{6} f$ on $[-2,2] \times[-2,2]$ in $a_{11}-a_{22}$ plane.

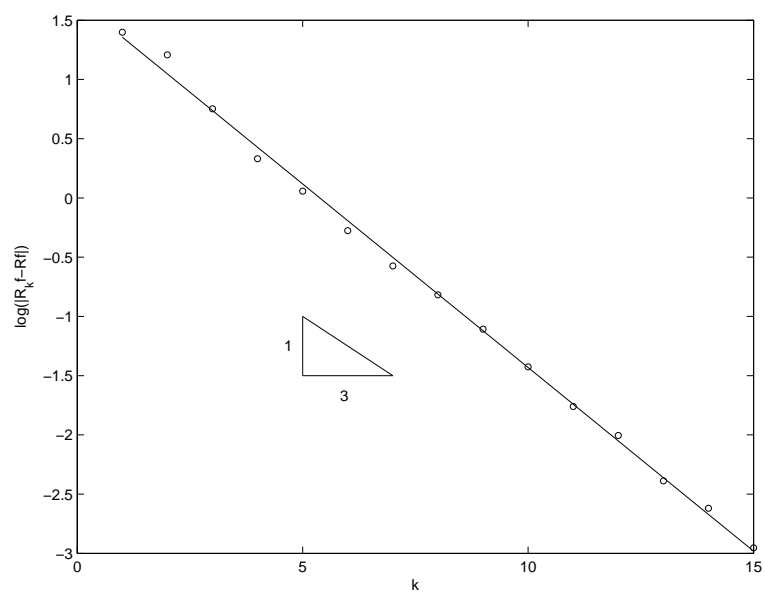

Figure 10. $\left\|R_{k} f-R f\right\|_{\infty}$ on $[-1,1] \times[-1,1]$ with $h=0.1$.

Figure 10 shows the convergence behavior of $R_{k}^{h} f$ with respect to $k$ for $h=0.1$ in the set $\mathcal{D}$ where $a_{12}=a_{21}=0$ and both $\left|a_{11}\right|$ and $\left|a_{22}\right|$ are no greater than 
1. In fact, our numerical experiments show that $R_{k}^{0.1} f(A) \approx R f(A)+46 \cdot 10^{-\frac{1}{3} k}$, which implies a superlinear convergence with respect to $k$.

\section{REFERENCES}

[1] Adranda, E. and Pedregal, P.(2001) On the computation of the rank-one convex hull of a function. SIAM J. Sci. Comput., 22(5), 1772-1790.

[2] Ball, J. M. and James, R. D.(1987) Fine phase mixtures as minimizers of energy. Arch. Rat. Mech. Anal., 100(1), 13-52.

[3] Ball, J. M. and James, R. D.(1992) Proposed experimental test of a theory of fine microstructure and the two-well problem. Phil. Trans. R. Soc. London 338A, 389-450.

[4] Chipot, M.(1999) The appearance of macrostructures in problems incompitable wells and their approach. Numer. Math., 83, 325-352.

[5] Dacorogna, B. (1989) Direct methods in the calculus of variations, Apllied Math. Sciences, vol. 78, Springer Verlag.

[6] Dolzmann, G. and Walkington, N. J.(1998) Bounds on the effective energy by rank-one convexification, in Enumath 97, H. -G. Bock, F. Brezzi, R. Glowinski, G. Kanschat, Y. A. Kuznetsov, J. Périaux, R. Rannacher, eds., 270-277.

[7] Dolzmann, G.(1999) Numerical computation of rank-one convex envelopes. SIAM J. Numer. Anal., 36, 1621-1635.

[8] Dolzmann, G. and Walkington, N. J.(2000) Estimates for numerical approximations of rank-one convex envelopes. Numer. Math., 85, 647-663.

[9] Kohn, R.V. and Strang, G. (1986), Optimal Design and relaxation of variational problems, I-III, Comm. Pure Appl. Math., 39, 113-137, 139-182, 353-377.

[10] Li, Z.-P.(2000) Finite order rank-one convex envelopes and computation of microstructures with laminates, BIT. Numer. Math., 40(4), 745-761.

[11] Li, Z.-P.(2004) Multiscale modelling and computation of microstructures in multi-well problems, Math. Mod. Meth. Appl. Sci., 9(14), 1343-1360. 